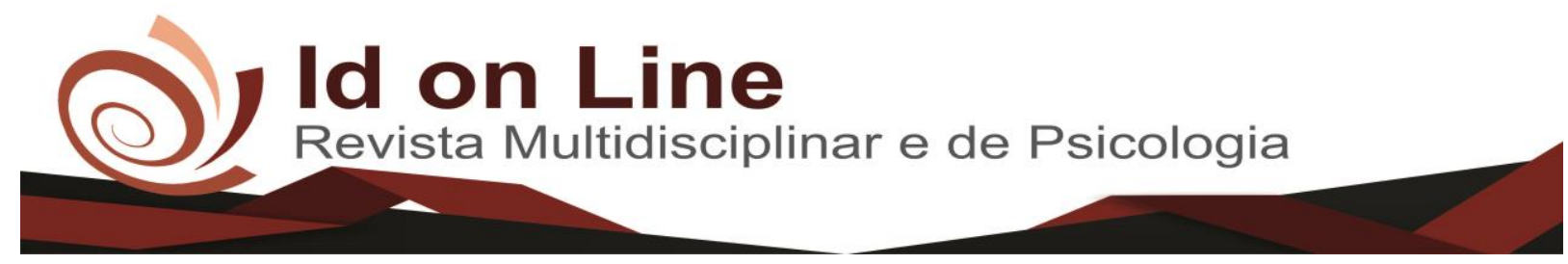

Artigo

\title{
Receios na Gestação de Alto Risco: Uma Análise da Percepção das Gestantes no Pré-Natal
}

\author{
Symara Abrantes Albuquerque de Oliveira Cabral ${ }^{1}$; Maria Carmem Batista de Alencar ${ }^{2}$; Larissa Araújo do Carmo ${ }^{3}$;
} Sylvio Elvis da Silva Barbosa ${ }^{4}$; Anny Caroline Costa Vieira Barros ${ }^{5}$; Jefferson Kleber Batista Barros ${ }^{6}$

Resumo: A gestação constitui-se de um evento carregado de sentimentos e capaz de marcar a vida da mulher de forma positiva ou negativa, o que, por si só, traz intensos receios, especialmente se tal evento vem acompanhado de processos patológicos que podem desencadear o receio do desfecho. Neste sentido, o presente trabalho objetivou analisar a percepção das gestantes quanto receios associados aos riscos de uma gestação acompanhada em prénatal de alto-risco. Foi desenvolvida uma pesquisa de campo, exploratória, prospectiva, com abordagem quantitativa e qualitativa dos dados, realizada em uma maternidade do sertão paraibano, com a população constituída por todas as gestantes admitidas no serviço obstétrico de alto risco de referência nos meses de maio e junho de 2017. A partir do estudo foi possível observar que a maioria das gestantes acolhidas no serviço não são consideradas de alto risco por não se enquadrarem nos parâmetros de admissão, e mesmo sendo bem orientadas carregam receios relativos ao nascimento do bebê, seja do momento do parto ou da vitalidade do concepto.

Palavras-chave: Cuidado Pré-Natal; Gravidez de Alto Risco; Medo.

\section{Fears in High Risk Pregnancy: an Analysis of the Perception of Pregnant Women in Prenatal}

Summary: Pregnancy is an event full of feelings and capable of marking a woman's life in a positive or negative way, which in itself causes intense fears, especially if such event is accompanied by pathological processes that can trigger the fear of the outcome. In this sense, the present study aimed to analyze the perception of pregnant women regarding fears associated with the risks of gestation accompanied in high-risk prenatal care. An exploratory, prospective, quantitative and qualitative data survey was carried out in a maternity hospital in the Sertão of Paraíba, with the population comprised of all pregnant women admitted to high-risk obstetric referral service in the months of May and June 2017. Based on the study, it was possible to observe that the majority of pregnant women in the service are not considered to be at high risk because they do not fit into the parameters of admission, and even if they are well-oriented they carry fears related to the birth of the baby, childbirth or the vitality of the concept.

Keywords: Prenatal Care; High Risk Pregnancy; Fear.

\footnotetext{
1 Graduação em Enfermagem pela Universidade Federal de Campina Grande. Especializações em: Saúde da Família, Obstetrícia, Gestão e Metodologias ativas. Mestrado multiprofissional em Sistemas Agroindustriais com ênfase na saúde. Doutoranda em Ciências da Saúde pela Santa Casa da Misericórdia de São Paulo. Contato: symara_abrantes@ hotmail.com;

${ }^{2}$ Mestrado Acadêmico em Sistemas Agroindustriais pela Universidade Federal de Campina Grande. Mestrado Profissional em Saúde Publica pela Universidad Tecnológica Intercontinental. Especialização em Preceptoria em Residência no SUS pelo Sírio Libanês. Especialização em Saúde Pública pela Faculdade São Francisco da Paraíba. Especialização em Saúde da Família pela Faculdade Integrada de Patos. Especialização em Enfermagem do Trabalho pela Faculdade Integrada de Patos. Graduação em Enfermagem pela Universidade Federal de Campina Grande. Docente da Graduação em Enfermagem da Faculdade São Francisco da Paraíba. Contato: carmemsjp@hotmail.com;

${ }^{3}$ Acadêmica do Curso de Graduação em Medicina pela Faculdade Santa Maria - FSM, Cajazeiras - PB. Possui Graduação em Enfermagem, pela Universidade Regional do Cariri - URCA;

${ }_{4}^{4}$ Acadêmico do Curso de Graduação em Medicina pela Faculdade Santa Maria (FSM), Cajazeiras - PB. Possui Graduação em Engenharia Metalúrgica pela Escola de Minas/Universidade Federal de Ouro Preto (2000). Mestre em Engenharia Ambiental - Uso e Conservação de Recursos Hídricos pela Universidade Federal de Ouro Preto.

${ }^{5}$ Graduada em Fisioterapia pela Faculdade de Ciências Aplicadas Doutor Leão Sampaio, Pós-graduada em Terapia Intensiva e Suporte Ventilatório para Faculdade Maurício de Nassau e pós-graduada em Políticas Públicas em Saúde da Família pela Universidade Regional do Cariri (URCA). Contato: anny_carol_15@hotmail.com;

${ }^{6}$ Graduação em Medicina pela Universidad Internacional Tres Fronteras - Cidade do Leste, Paraguai.
} 


\section{Introdução}

A decisão de tentar gerar um concepto muitas vezes passa por uma decisão em conjunto, ou seja, é algo de estrema importância na relação entre o homem e a mulher. Entretanto, existem algumas situações nas quais a gravidez acontece de maneira inesperada, principalmente, naquelas mulheres que não tiveram uma instrução adequada sobre os métodos de proteção existentes.

A gravidez é um evento capaz de consolidar a mudança de um ciclo de vida a outro, é o momento no qual ela sente-se realmente plena, sente-se mulher. É um momento de mudanças várias e intensas, capazes de desencadear sentimentos positivos ou negativos (SOUSA; COSTA; PINON, 2010).

Independentemente da forma como se iniciou a gestação, a mulher grávida, com o passar dos meses, poderá enfrentar várias expectativas e anseios, frutos das alterações fisiológicas mediadas por condições físicas ou hormonais, a nível corporal, de ordem psíquica, da expectativa de futuro sobre o concepto e até mesmo medo do ato do parto, principalmente nas primíparas. Deste modo, a gravidez é vista pelas mulheres como um momento de transição extremamente significativo, que exige adaptações diversas.

Tomando por base os aspectos psíquicos, suas complicações podem ser geradoras e ansiedade e depressão, neste sentido, estudos realizados por Kliemann; Böing; Crepaldi (2017), no qual realizou-se uma revisão sistemática de artigos empíricos, foram definidos como fatores de risco para ansiedade e depressão na gestação: aspectos cognitivos; rede social de apoio ineficaz; enfrentamento da violência; intercorrências físicas maternas e/ou fetais; eventos estressores na gestação; relacionamento conjugal e familiar conflituoso; saúde mental (histórico de depressão e/ou transtorno de ansiedade, transtornos mentais, níveis altos de ansiedade, e alta percepção de estresse); uso de substâncias psicoativas na gestação; fatores socioeconômicos (desemprego ou vulnerabilidade econômica); estrutura familiar (ter dois ou mais filhos ou não ter filhos); idade materna (extremos); baixo nível educacional; e gravidez não planejada ou indesejada.

Importante considerar que, de acordo com Montenegro (2017), as mulheres que apresentam determinadas complicações no período gestacional estão mais propensas ao desenvolvimento de doenças crônicas ao longo da vida, sendo a gravidez, citando que a préeclâmpsia na gravidez pode determinar risco para doença cardiovascular futura, e ainda que 
mulheres acometidas por diabetes gestacional, tem grandes chances de tornarem-se diabéticas tipo 2.

Neste sentido, é importante que o profissional responsável pelo acompanhamento prénatal tenha pleno conhecimento das alterações fisiológicas da gestação, de modo que, conhecendo o que é fisiológico tem-se a capacidade de identificação dos processos patológicos, a mulher precisa adaptar-se as alterações fisiológicas da gestação e quando o processo adaptativo é deficiente observa-se a instalação do processo patológico, de modo que, a incapacidade da mulher para se adaptar às mudanças e exigências da gravidez pode afetar a saúde física e mental e parece influenciar, de igual forma, a saúde do concepto (ZUGAIB, 2016).

Neste sentido, mesmo que se faça um bom acompanhamento pré-natal, conforme estabelecido pelo Ministério da Saúde, a gestação poderá acarretar algumas complicações obstétricas associadas aos fatores de risco que podem ter consequências negativas para o binômio mãe/filho e no próprio desfecho gestacional (ARAUJO; PEREIRA; KAC, 2007). Importante ressaltar que os desfechos desfavoráveis muitas vezes são disseminados na comunidade de forma errônea, propagando informações incoerentes, capazes de gerar ainda mais dúvidas e receios para as mulheres grávidas.

Deste modo, é fundamental oferecer um acompanhamento singularizado para cada gestante durante o pré-natal, levando em consideração os aspectos socioeconômicos, os obstétricos, anseios, estilo de vida, aspectos nutricionais, dentre outros, de modo a consolidar um vínculo de confiança entre a gestante e os profissionais envolvidos para melhor ajudá-las a enfrentar as adversidades que poderão sugerir durante todo esse período, especialmente no contexto do pré-natal de alto risco.

Diante de tais preceitos, a presente pesquisa objetivou analisar a percepção das gestantes quanto a receios associados aos riscos de uma gestação acompanhada em pré-natal de altorisco. Além de que, foram ainda considerados objetivos: identificar as principais causas que justificam o pré-natal de alto risco; analisar percepções errôneas que podem vir a fragilizar a gestante; avaliar a qualidade das informações adquiridas no pré-natal quanto complicações associadas a gestação; estabelecer parâmetros de melhoria das informações prestadas no prénatal. 


\section{Material e Método}

O presente trabalho trata-se de uma pesquisa de campo, exploratória, prospectiva, com abordagem quantitativa e qualitativa dos dados, realizada em uma maternidade do sertão paraibano. O serviço de pré-natal de alto risco é realizado no Banco de Leite da maternidade e consolida-se como um serviço regionalizado e que atende aproximadamente quinhentas gestantes por mês.

A população do estudo foi constituída por todas as gestantes admitidas no serviço obstétrico de alto risco de referência no período estabelecido para pesquisa, admitindo-se por critérios de inclusão: gestantes acompanhadas no pré-natal de alto risco. Já como critérios excludentes tem-se as gestantes que não se enquadram nos critérios de inclusão e as que não aceitaram participar da pesquisa.

Foi utilizado como instrumento de coleta de dados um roteiro de entrevista semiestruturado, e a coleta foi realizada no próprio espaço, utilizando o momento de espera para o atendimento pré-natal de alto risco.

Os dados foram consolidados diante da proposta de análise de conteúdo de Bardin (2009) e categorizados estatisticamente, dispostos em quadros, tabelas e gráficos para melhor compreensão, considera-se válida a amostragem diante da utilização apenas uma variável: gestante participante do pré-natal de alto risco.

A pesquisa foi submetida a Plataforma Brasil e aprovada pelo Comitê de Ética da Faculdade Santa Maria através de parecer número 2.069.611.

\section{Resultados e Discussões}

A pesquisa foi realizada entre maio e junho de 2017, participaram do estudo trinta e uma (31) gestantes, configurando $89 \%$ das gestantes acompanhadas no serviço no período. À priori, importante considerar a idade das gestantes, conforme figura 01: 
Figura 01: idade das gestantes participantes do estudo.

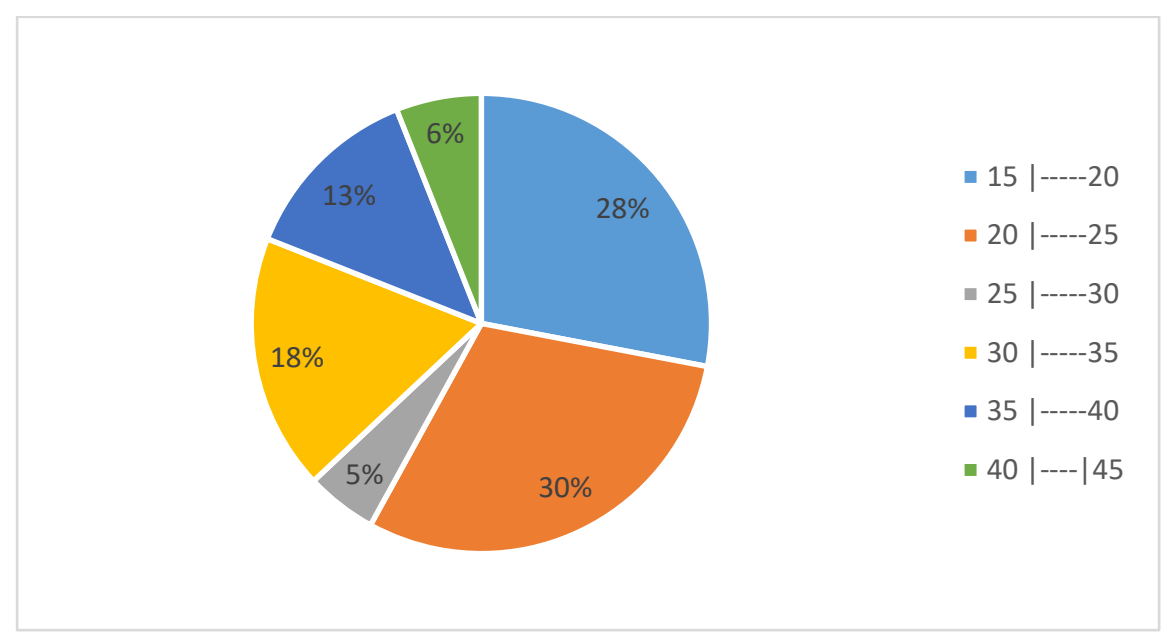

Fonte: dados da pesquisa, 2017.

Tem-se como participantes da pesquisa gestantes com média de vinte e sete vírgula três $(27,3)$ anos de idade, com extremos situados nos dezesseis (16) e quarenta e três (43) anos de idade. Tomando por base Montenegro (2017, p.21) "as mulheres são mais férteis entre 15 e 30 anos. É considerada "idosa" a grávida acima de 35 anos; no entanto, a definição de idade materna avançada na literatura mais recente foi elevada para 40 a 45 anos". Já considerando o Protocolo da Atenção Básica: Saúde das Mulheres do Ministério da Saúde (2016), a idade materna menor que quinze (15) e maior que trinta e cinco (35) anos configura-se fator de risco indicativo de realização do pré-natal de baixo risco. Deste modo, as idades das participantes do estudo correlacionam-se com as idades preconizadas na literatura atual.

Importante considerar que todas as gestantes acompanhadas no pré-natal de alto risco continuam o pré-natal na rede básica, sendo que apenas uma (01) delas refere realização do prénatal na rede particular de assistência.

Tomando por base os antecedentes obstétricos observou-se que a maioria das participantes eram múltiparas (65\%), dessas, 55\% foram acompanhadas em gestação anterior em pré-natal de baixo risco e apenas $10 \%$ em pré-natal de alto risco, considerando como causas de encaminhamento: descolamento de placenta associado a pré-eclâmpsia e infecção do trato urinário, sangramento não determinado, e pré-eclâmpsia. 
Para determinar as situações que demandaram acompanhamento no pré-natal de alto risco, correlacionamos as condições a idade gestacional para melhor compreensão, conforme figura 02 .

Figura 02: situações que demandaram acompanhamento de alto risco correlacionado a idade gestacional.

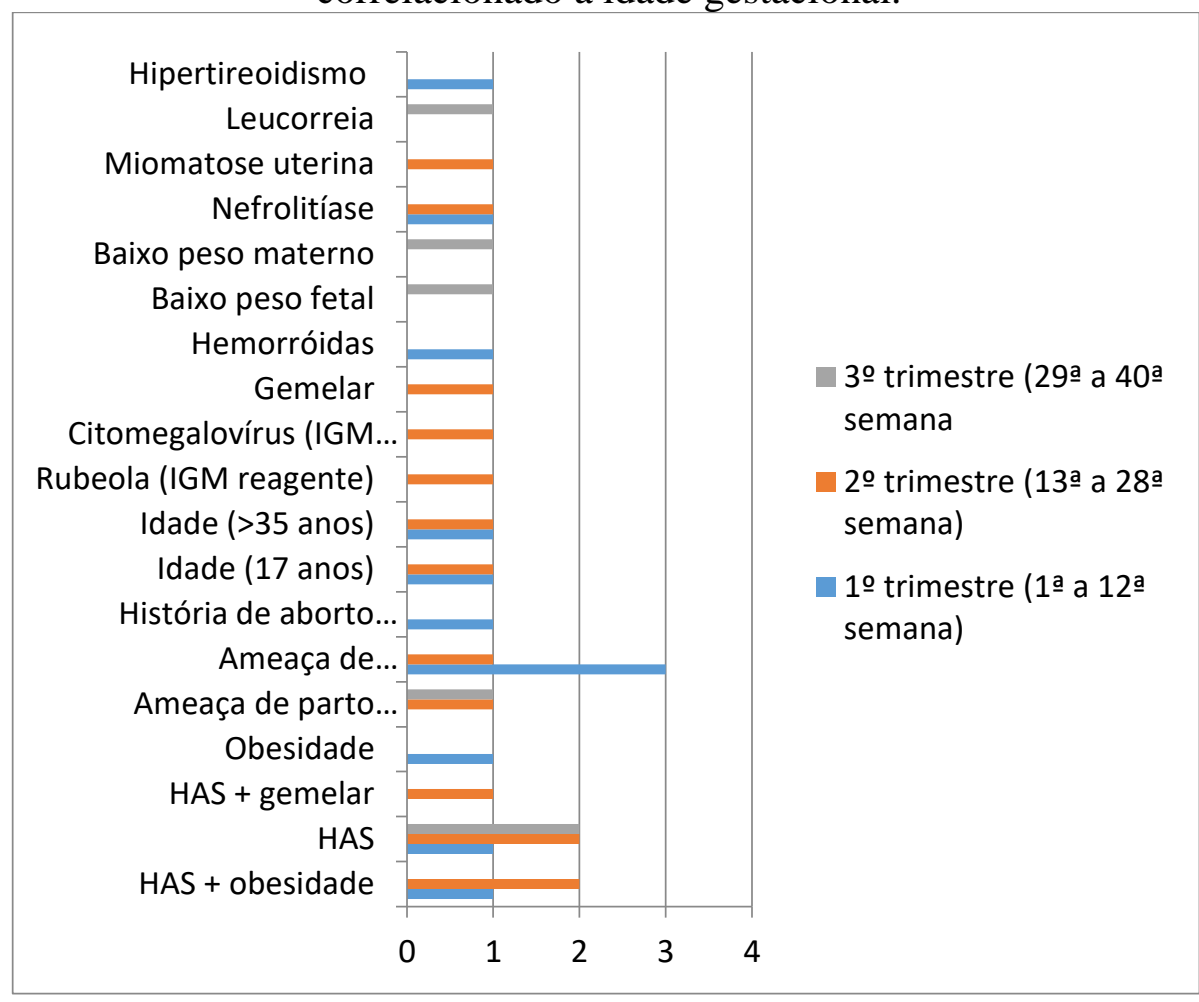

Fonte: dados da pesquisa, 2017.

Importante considerar que de acordo com Brasil (2016, p.34), são considerados fatores de risco indicativos de encaminhamento ao pré-natal de alto risco:

\section{FATORES RELACIONADOS ÀS CONDIÇÕES PRÉVIAS}

- Cardiopatias.

- Pneumopatias graves (incluindo asma brônquica não controlada).

- Nefropatias graves (como insuficiência renal crônica e em casos de transplantados).

- Endocrinopatias (especialmente diabetes mellitus, hipotireoidismo e hipertireoidismo).

- Doenças hematológicas (inclusive doença falciforme e talassemia).

- Doenças neurológicas (como epilepsia).

- Doenças psiquiátricas que necessitam de acompanhamento (psicoses, depressão grave etc.).

- Doenças autoimunes (lúpus eritematoso sistêmico, outras colagenoses).

- Alterações genéticas maternas.

- Antecedente de trombose venosa profunda ou embolia pulmonar. 
- Ginecopatias (malformação uterina, tumores anexiais e outras).

- Portadoras de doenças infecciosas como hepatites, toxoplasmose, infecção pelo HIV, sífilis terciária (USG com malformação fetal) e outras ISTs (condiloma).

- Hanseníase.

- Tuberculose.

- Anemia grave (hemoglobina < 8).

- Isoimunização Rh.

- Qualquer patologia clínica que necessite de acompanhamento especializado.

\section{FATORES RELACIONADOS À HISTÓRIA REPRODUTIVA ANTERIOR}

- Morte intrauterina ou perinatal em gestação anterior, principalmente se for de causa desconhecida.

- Abortamento habitual (duas ou mais perdas precoces consecutivas).

- Esterilidade/infertilidade.

- História prévia de doença hipertensiva da gestação, com mau resultado obstétrico e/ou perinatal (interrupção prematura da gestação, morte fetal intrauterina, síndrome HELLP, eclâmpsia, internação da mãe em UTI).

\section{FATORES RELACIONADOS À GRAVIDEZ ATUAL}

- Restrição do crescimento intrauterino.

- Polidrâmnio ou oligodrâmnio.

- Gemelaridade.

- Malformações fetais ou arritmia fetal.

- Evidência laboratorial de proteinúria.

- Diabetes mellitus gestacional.

- Desnutrição materna severa.

- Obesidade mórbida ou baixo peso (nestes casos, deve-se encaminhar a gestante para avaliação nutricional).

- NIC III.

- Alta suspeita clínica de câncer de mama ou mamografia com Bi-RADS III ou mais.

- Distúrbios hipertensivos da gestação (hipertensão crônica preexistente, hipertensão gestacional ou transitória).

- Infecção urinária de repetição ou dois ou mais episódios de pielonefrite (toda gestante com pielonefrite deve ser inicialmente encaminhada ao hospital de referência para avaliação).

- Anemia grave ou não responsiva a 30-60 dias de tratamento com sulfato ferroso.

- Portadoras de doenças infecciosas como hepatites, toxoplasmose, infecção pelo HIV, sífilis terciária (USG com

malformação fetal) e outras IST (infecções sexualmente transmissíveis, como o condiloma), quando não há suporte na unidade básica.

- Infecções como a rubéola e a citomegalovirose adquiridas na gestação atual.

- Adolescentes com fatores de risco psicossocial.

Tomando por base os critérios de inclusão de gestantes nos serviços de pré-natal de alto risco, é possível identificar no rol das situações que demandaram acompanhamento de alto risco uma maior incidência no grupo de doenças na gravidez atual e das intercorrências clínicas. É possível ainda observar na figura 02, e preocupante se faz constatar, que $61 \%$ das gestantes poderiam ser assistidas no pré-natal de risco habitual na Unidade de Saúde da Família. 
Importante considerar ainda que há uma maior incidência de gestantes com hipertensão arterial sistêmica, associada, ou não a outros fatores, resultado esse que assemelha-se a recente estudo desenvolvido por Jantsch; et al. (2017).

Partindo agora para a busca pela contemplação dos objetivos propostos e tomando por base o questionamento: como você de sentiu ao receber a notícia que iria ser acompanhada no pré-natal de alto risco? Têm-se as respostas agrupadas por análise de conteúdo dispostas na figura 03 .

Figura 03: Respostas frente ao questionamento: como você se sentiu ao receber a notícia que iria ser acompanhada no pré-natal de alto risco?

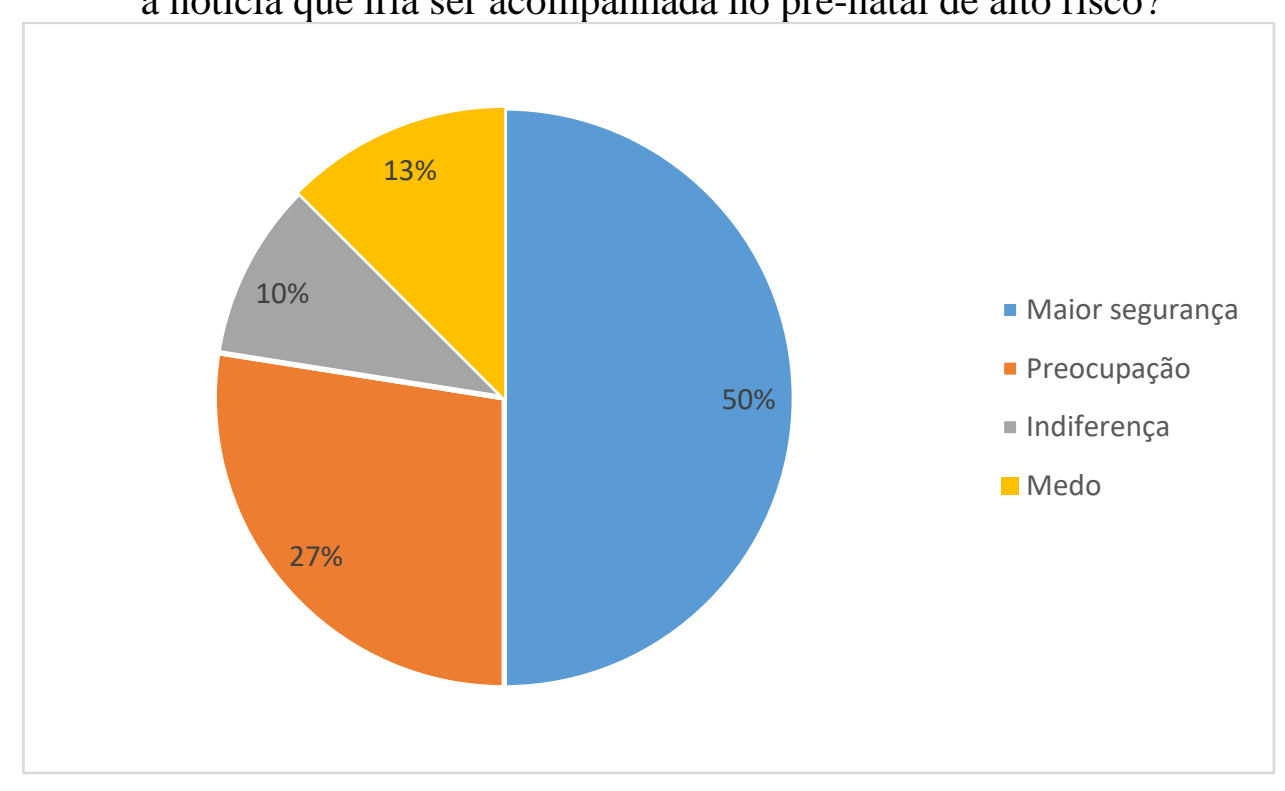

Fonte: dados da pesquisa, 2017.

Os dados da figura 03 revelam que a maioria (42\%) das gestantes refere o sentimento de preocupação, e apontaram a falta de informação sobre a patologia como principal fonte de tal sentimento. Dentre as gestantes que referiram sentir maior segurança $(23 \%)$ referiram que o serviço oferece uma melhor qualidade e maior qualidade das informações. Já dentre as que revelam medo (19\%), relacionam ao desfecho da gestação, a própria saúde e quando as complicações que, porventura, podem ocorrer. Interessante observar que algumas gestantes revelam indiferença (16\%), dentre as quais revelam que sabem que não deveriam ser atendidas no alto risco, por isso não se preocupavam, e apenas uma delas revela o desejo de realizar cesárea e considera o serviço como um meio para conseguir sem objetivo. 
Sabendo que a falta de conhecimentos sobre a situação atual de saúde ou condição atual de cuidado são fatores preponderantes para despertar o sentimento de preocupação, as gestantes foram questionadas sobre qual o conhecimento que tem sobre o que levou ao acompanhamento de alto risco. Neste caso, $65 \%$ das gestantes referem não ter conhecimento algum sobre o fator preponderante para o encaminhamento ao alto risco. O princípio da autonomia defende o direito da gestante em ter conhecimento dos procedimentos a que estão sujeitas, bem como fazer uma escolha sobre o tipo de parto. Trata-se de algo que envolve muita discussão entre os membros da equipe de saúde. Em geral, a gestante não participa de tal discussão, apenas é informada a posteriori. Em muitos dos casos não lhes é explicado a dimensão do risco a que se expõe (FERRARI, 2009).

Buscando melhor compreensão acerca das informações e orientações prestadas, as gestantes foram questionadas sobre que momento e por qem sentiu-se mais orientada, de modo que apenas uma gestante revelou não ter tido nenhuma orientação, dentre as demais, todas referiram que desde o início do pré-natal, $75 \%$ referem que pela enfermeira, $15 \%$ pelo médico, $5 \%$ pelo médico do alto risco, e $5 \%$ pelos grupos de gestante que participavam.

As gestantes foram questionadas ainda sobre os receios que permeiam a gestação, de modo que os dados foram dispostos na figura 04.

Figura 04: Respostas frente ao questionamento: quais os receios que você tem quanto a sua gestação?
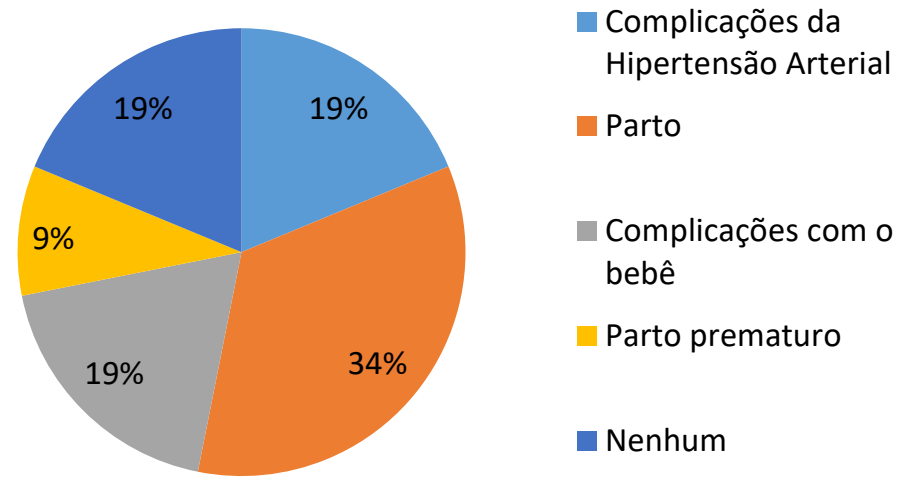

Nenhum

Fonte: dados da pesquisa, 2017. 
É possível observar na figura 04 que a maioria das gestantes demostra receio quanto ao parto (34\%), talvez pelo déficit de informações concisas sobre esse momento. Já em correlação com as patologias que demandaram o acompanhamento no pré-natal de alto risco, talvez como uma própria resposta, tem-se o receio de complicações da HAS e complicações com o bebê (19\% ambos), do parto prematuro (9\%).

A sensibilização da equipe de saúde com relação à importância da forma de assistência, levando-se em conta os medos e autossugestões da mulher naquele momento para aquela mulher é fundamental. Simples atitudes de acolhimento e escuta empática são eficazes e, tendem a influenciar positivamente na assistência à mãe, principalmente quando primípara (MOURA et al., 2007).

Buscando melhor complementaridade das informações e prevendo que o parto seria um dos receios demonstrados pelas gestantes, estas foram questionadas sobre a via de parto que preferiam e quais as orientações proferidas no pré-natal, de modo que $60 \%$ foram orientadas quanto ao parto normal a partir dos princípios da melhor recuperação e menor risco. E, por fim, quando as perspectivas para o nascimento do filho, unânime é o desejo de que tudo ocorra bem e que a o bebê nasça saudável.

\section{Conclusões}

Tomando por base os objetivos propostos, o presente trabalho permitiu analisar a percepção das gestantes quanto aos receios associados aos riscos de uma gestação acompanhada em pré-natal de alto risco, constatando-se que parte das gestantes encaminhadas ao pré-natal de alto risco poderiam ser acompanhadas no risco habitual, minimizando as demandas de locomoção para longe de sua área de abrangência e ainda minimizando a possibilidade de sentimentos negativos de preocupação e medo. Quanto às causas que demandaram o acompanhamento de alto risco, é considerável a ocorrência de hipertensão, seja crônica ou específica da gestação, acompanhada ou não de fatores de risco, como a obesidade.

Importante considerar que há déficit considerável de informações no transcorrer do prénatal, fator também preponderante para sentimentos negativos e ainda para anseios que poderiam ser evitados, como o receio do parto, que, por sua 
vez, deveria ser considerado um evento de prazer e realização. É, de tal modo, notória a necessidade de melhora no contexto do pré-natal de risco habitual, seja pelo melhor critério de encaminhamento ao alto risco, seja pela melhoria na qualidade das informações prestadas, garantindo, assim, melhor qualidade de vida e maior probabilidade de compreensão da gravidez como um evento positivo por parte da mulher gestante.

Os profissionais de saúde precisam compreender que a mulher gestante precisa de uma atenção qualificada, capaz de minimizar os receios desse período tão carregado de necessidade de adaptações psicológicas, fisiológicas, endócrinas ou sociais. É preciso compreender que a gestação por si só não deve ser considerada como um processo patológico, ser capaz de identificar quando os processos adaptativos não ocorrem de modo adequado e ainda quando patologias de base são agravadas para, assim, proceder a um acompanhamento de qualidade que garanta melhoria também nos desfechos insatisfatórios, como a morbimortalidade materna, fetal ou neonatal.

\section{Referências}

ARAUJO, D. M. R.; PEREIRA, N. L.; KAC, G. Ansiedade na gestação, prematuridade e baixo peso ao nascer: uma revisão sistemática da literatura. Cadernos de Saúde Pública, 2007; 23: 747-756.

BARDIN, L. Análise de Conteúdo. Lisboa, Portugal; Edições 70, LDA, 2009.

BRASIL. Ministério da Saúde. Protocolos da Atenção Básica: Saúde das Mulheres / Ministério da Saúde, Instituto Sírio-Libanês de Ensino e Pesquisa - Brasília: Ministério da Saúde, 2016.

FERRARI, J. A autonomia da gestante e o direito pela cesariana a pedido. 2009. Disponível em: <http://revistabioetica.cfm.org.br/index.php/revista_bioetica/article/ viewFile/512/513>. Acesso em: 20 mar. 2016.

JANTSCH, P.F.; et al. Principais características das gestantes de alto risco da região central do Rio Grande do Sul. Revista Destaques Acadêmicos, Lajeado, v. 9, n. 3, 2017.

KLIEMANN, A.; BÖING, E.; CREPALDI, M.A. Fatores de risco para ansiedade e depressão na gestação: Revisão sistemática de artigos empíricos. Mudanças - Psicologia da Saúde, 25 (2), Jul.-Dez. 2017. 
MONTENEGRO, C.A.B. Rezende obstetrícia. 13. ed. - Rio de Janeiro : Guanabara Koogan, 2017.

MOURA, F. M. J. et al. A humanização e a assistência de enfermagem ao parto normal. Rev. bras. enferm., v.60, n.4, 2007. p.452-455. Disponível em: <http://dx.doi. org/10.1590/S0034$71672007000400018>$. Acesso em: 7 jun. 2016.

SOUSA, L. B. de ; COSTA, E. S.; PINON, G. M. B. Alterações fisiológicas na percepção de mulheres durante a gestação. Rev. Rene, Fortaleza, v. 11, n. 2, p. 86-93, abr./jun. 2010.

SOUZA, B. L. P. et al. Humanização no atendimento ao pré-natal. Instituto Federal de Educação, Ciência e Tecnologia do Sul de Minas - campus Muzambinho - pólo da rede unidade de ensino Capetinga. Trabalho de monografia, 2010.

ZUGAIB, M. Zugaib obstetrícia. 3. ed. - Barueri, SP: Manole, 2016.

\section{Como citar este artigo (Formato ABNT):}

CABRAL, Symara Abrantes Albuquerque de Oliveira; ALENCAR, Maria Carmem Batista de; CARMO, Larissa Araújo do; BARBOSA, Sylvio Elvis da Silva; BARROS, Anny Caroline Costa Vieira; BARROS, Jefferson Kleber Batista. Receios na Gestação de Alto Risco: Uma Análise da Percepção das Gestantes no Pré-Natal. Id on Line Rev.Mult. Psic., 2018, vol.12, n.40, p.151- 162. ISSN: 19811179 .

Recebido: $16 / 02 / 2018$

Aceito 24/02/2018 